\title{
Designing Visceral, Behavioural and Reflective Products
}

\author{
Mersha Aftab ${ }^{1} \cdot$ Helen Agustin Rusli ${ }^{1}$
}

Received: 8 December 2016/Revised: 30 May 2017/Accepted: 4 July 2017 /Published online: 1 August 2017

(c) The Author(s) 2017. This article is an open access publication

\begin{abstract}
Designers and manufacturers often see consumption as the primary objective of a product - with implications such as discarded products, obsolete wastes, and ecological degradation. The paper aims to find the answer to the question, how emotional design can adapt the discarded and undesirable products into something valuable in a long term? This paper presents a framework combining Chapman's theory and Norman's theory on three levels of emotional design to highlight what long lasting connection with products entails. A design approach is presented combing the Wabi Sabi philosophy that promotes the celebration of decay and damage. This is used as one of the design principles for the experiments conducted on discarded products. Through constant user interaction before, during and after the experiments the evaluation of design as an agent of transformation is done. The user conducted the evaluation based on the Kansei elements of looks, sound, smell, and feel of the product. The experiments confirmed that a long-term value is only achieved through redesigning and reconstructing the perception of people towards products on a reflective level, rather than the visceral and behavioural elements of the product. The research found attachment to the visceral and behavioural elements of a product instead of an emotional one was causing users to discard products faster than required. The research indicated that many people, including designers and manufacturers, are unconsciously focusing on usability (behavioural level) and physical look (visceral level) of a product that are easily replaced, than on a meaningful way
\end{abstract}

Mersha Aftab

m.aftab@northumbria.ac.uk

1 Northumbria School of Design, Northumbria University, Newcastle Upon Tyne NE1 8ST, UK (reflective level) to create and maintain long-lasting emotions. The research concluded with a proposition towards digitization of products which could perhaps be an all round solution to make products more appropriate to human emotions. Digitization could give products the ability to capture, store and then communicate the stories, journey and memories back, in order to empower people to understand the value of longer-term use of products.

Keywords Research through design - Emotional design . Kansei design · Recycling · Upcycling · Product design

\section{Introduction}

It is believed that the rapid increase in the pace of manufacturing influences the current consumer culture, where short-usage and discarded products emerge. Designers and manufacturers often see consumption as the primary objective of a product, where discarded products, obsolete wastes, and ecological degradation are the implications. Capitalism and mass production dominates the economy by efficient and cheap production that allows people to easily afford new products with a better value for money than repairing or preserving the existing products, and the stories that these products help create.

Kwan [1] pointed out that many products are designed with less durability for people to keep consuming. People do not get emotionally attached to products because they are excluded from the design and manufacturing processes. Their lack of empathy inflicts a long lasting relationship between people and products. Many sustainable and adaptable design solutions, such as recycling/up-cycling, have been explored for the given problem, but researchers concluded that neither are the optimal solution [2]. Many 
techniques of recycling and up-cycling are mainly concerned with functional aspects, i.e., maximizing a products main purpose without considering its desirability. Jordan [3] explained that it is inevitable for the people to get used to usable products and want something more. He concludes that, "products that bring not only functional benefits but also emotional ones."

The current investigation takes a research through design approach [4] to identify people's responses to redesigned discarded/unused products, in order to make them more desirable. The investigation questions many current trends and proposes new ways of looking at the user-product relationship at functional, behavioural and reflective level. Additionally, it provokes a discussion around a new role digital technology could play in making products of tomorrow more reflective, which the authors find a unique but necessary agenda for the future.

The paper reviews the implication of the exponentially growing manufacturing industry has on the people and their long term use of products. Whilst conducting a few design led experimentations on discarded or unwanted products, the paper demonstrates the need for a more human-centered and reflective approach towards product design and it's manufacturing, so that products could connect with its users at an emotional and reflective level. The research question that this paper is addressing is, how emotional design can adapt the discarded and undesirable products into something valuable in a long term?

The remaining of this paper is structured as follows.

Section 2 reviews key theories from Chapman [5] and Norman [6] to identify the key dimensions for emotional design. Section 3 illustrates the research through design methodology by giving a detailed description of the different stages of the research and the importance of users for the overall research methodology. Section 4 discusses the final findings obtained from the user research. Section 5 demonstrates the design experiments and identifies how each of the products was changed to imbibe the visceral, behavioural and reflective aspects of the emotional design. Section 6 demonstrates the ways the outcomes of the experiments were evaluated. Section 7 concludes the paper by identifying the need to think for products to be more reflective in order to have a longer-term emotional connection with their user.

\section{Development of a Framework for Successful Emotional Design for a Long-term Use}

In order to find a possible explanation on factors enabling a long-lasting emotional relationship between products and users, we first developed a framework. This was done by reviewing and comparing three key theories from Chapman
[5] and Norman [6]. Later, this framework was used to find a solution to reconstruct a new relationship between the users and the products.

Emotions have a vital role in human's ability to understand and create meaning of the world. Chapman [5] and Norman [6] pointed out that emotional design can be the solution to build a strong emotional attachment and empathy between people and products which can also influence people's life - the way people think, feel, and act. Norman [2] concluded that a successful design excels in three levels of emotional design - visceral level (appearance and pleasure to see), behavioural level (usability and performance), and reflective level (the meaning, self-image, and message of a product).

Firstly, visceral is the most basic level that creates immediate response and is associated with appearance and pleasure to see a well-designed product. The visceral quality of a product establishes the first emotional connection between the user and the product.

Secondly, behavioural level is associated with the whole experience of using a product - usability, understanding, physical feel, and performance - where appearance does not really matter.

Lastly, reflective being the highest level of emotional state of design; it is about the message, the culture, selfimage and the meaning of a product that is communicated to others. However, emotional design should be able to sustain people's empathy towards products in a long term.

Chapman [5] critically observed that, "some products are discarded before they are physically worn out or are technically superseded because their design is out of fashion or inappropriate to changed circumstances." $\mathrm{He}$ pointed out everything in the world continually changes, and so does the nature of products and people's empathy. Empathy on the other hand has a lifespan, and product waste as the symptom of expired empathy is caused by the lack of people's emotional attachment to their products.

\subsection{Reflective Products}

Products should create a long-term relation through a narrative experience of user-reflection to the past and contemplation to the future - produced by the meaning that is personally given by people to products. Juniper [7] introduced Wabi Sabi, a Japanese philosophy that sees the value of product as a journey of narrative experiences. Wabi Sabi accepts the lifecycle - the nature of growth, age, and patina of products, where the quality of products is not seen by its performance, functions, or objectivity, rather it is seen through it's journey and stories behind a product. Juniper [7] acknowledged "such qualities as impermanence, humility, asymmetry, and imperfection." The 
cornerstone of Wabi Sabi philosophy is personal responsibility of creating meaning to products.

Norman [6] pointed that "there is on other distinction among the levels: time. The visceral and behavioural levels are about "now," the feelings and experiences while actually seeing or using the product. But the reflective level extends much longer - through reflection you remember the past and contemplate the future. Reflective design, therefore, is about long-term relations, about the feelings of satisfaction produced by owning, displaying, and using the product." Beyond the design of an object, there is personal component that no designers or manufacturers can provide. Reflective attachment is personally given by users and more likely to produce emotional durability. Emotional attachment is not determined by a certain condition of products, but it defined by willingness of users to perceive, reflect, and give meanings to products.

There are always reasons for meaningful product design that reach a reflective level of emotional attachment to be maintained by users in longer time, transcend the fluctuation of behavioural and visceral design changes. The lack of usability (behavioural level) can be repaired and improved. Physical decays, defects and damages (visceral level) of products can be seen as a part that has richer experience, rather than the 'less valuable' parts.

\subsection{Behavioural Products}

Brand [8] in How Buildings Learn confirmed that even buildings change, "First we shape our buildings, then they shape us, then we shape them again - ad infinitum. Function reforms form, perpetually." The needs, preferences, and experiences of use from one building occupants to another will be different over the time, so does consumers of products. Different consumers may modify the structure to meet their new needs with their personal value and meaning. This continual of use happens with adaptive reuse of the same product.

Products should be physically robust and adaptable enough for people to use in a long term and help repurposing discarded products with the same or different function. However, Norman [6] suggested in order to make them pleasurable to use in a long term, products should be physically robust from the beginning of design process with materials that can gracefully age. A long lasting usability can be determined by sustainable, good performance and good physical feel of a product.

\subsection{Visceral Products}

Products should be visceral by high standard aesthetic quality that can be cherished in a long time. At a visceral level, Walker [9] observed, "many contemporary products rely on shiny, highly polished, new surfaces for their visual appeal". Aesthetics of modern design often defy the natural aging effects of time, which create unsustainable empathy when the product is physically decayed through times.

As an aesthetic philosophy, Wabi Sabi suggests the beauty of a product comes from intuition and non-intellectual feeling. It does not view the world in "black and white" perception, it has no duality - no beauty, no ugliness. "Our ideas of what represents beauty and ugliness are based mainly on learned assumptions about the items that we perceive in our own separate worlds" [7]. The reality of physical impermanence is acknowledged, natural aging effects are embraced, and the transformations are used as integral part of the whole design without forcing too many interventions. Wabi Sabi accepts the natural growth of product through times and physical changes, and can reduce continual dissatisfaction of beauty and acquire a longer empathy.

Visceral design of products can play an important role to visually deliver a message to people. Refs. [10] and [11] enhanced colour as the most obvious points of attraction to catch audience's attention. In fact, colour can be one of the most powerful design elements to effectively communicate messages to people in a busy visual environment [11]. At a psychological level, Ref. [11] pointed out that colour can be a primary motivator for altering behaviour. Kopec [12] agreed that colour is associated with meaning and evokes emotion that will impact people's conscious and subconscious mind. However, Ching and Binggeli [13] carefully uncover that different people may emotionally react to colour diversely, driven by different experiences, genders, cultures, and political views. The various perception, interpretation and reaction of people to certain colours require experiment and further validation in this project.

The importance of eyesight, taste, touch and smell in creating a deeper connection between products and the users are well documented in the Kansei Engineering and design literature [14]. Gentner, et al. [15]. highlight the application of Kansei methodology at the beginning of new concept development in Toyota. Their investigation concluded that three steps namely understand, create and assess, all within the premise of aforementioned sensory receptors, are a necessity to inform the new product development process, in order to make the products more human centric. As a result of the application of Kansei methodologies, many organisations, such as Volvo, Toyota, Nissan, have been able to create lean and human centric manufacturing processes which eliminate waste and create products that connect with its users on an emotional level. However, Kansei's application to products such as furniture (specifically inexpensive products) is still unknown. Whilst current manufacturing approaches, specifically for mass manufacturing, allows products to be 


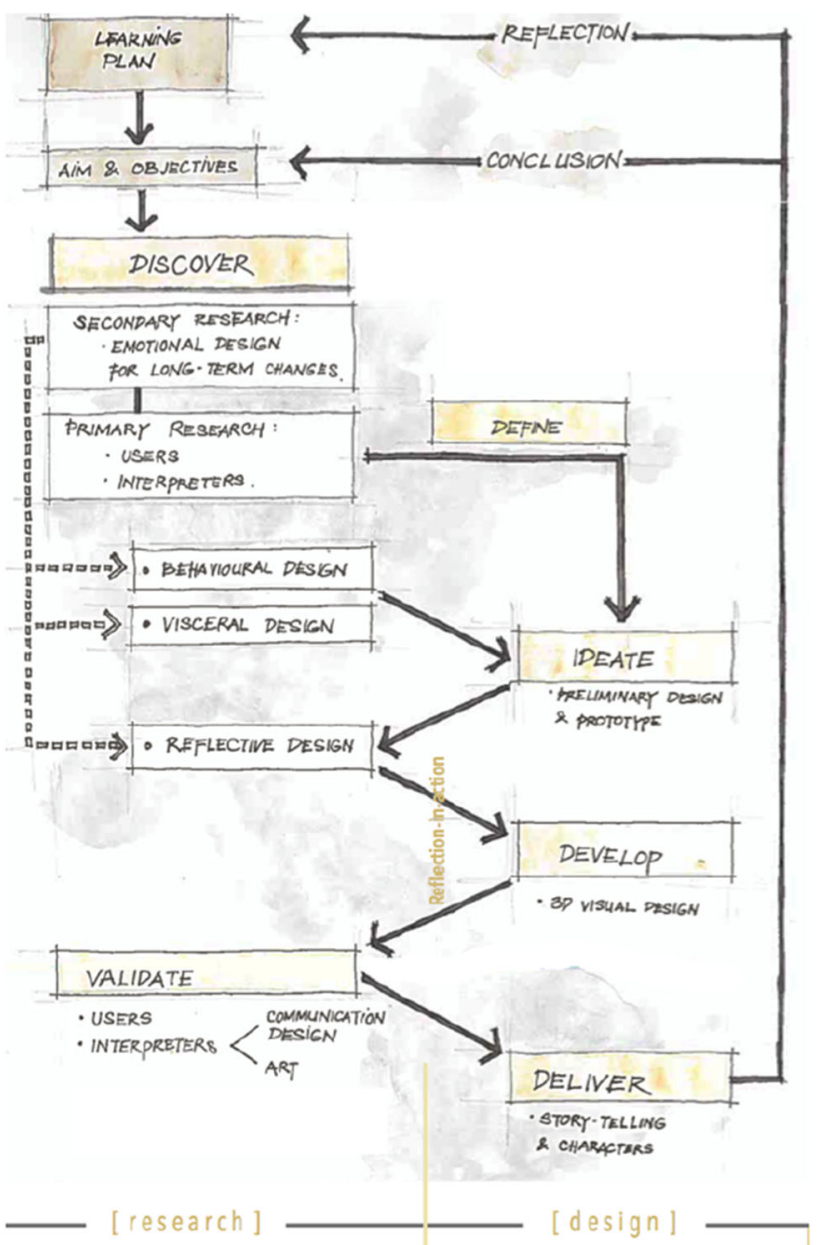

Figure 1 Research through design process

visceral, these products lack creating long-term experiences due to their short life span and lack of establishing an emotional connection.

Through combining Chapman's [5] and Norman's [6] theory, a framework with elements for successful emotional design for a long-term use emerges (Appendix 1).

This investigation set out to determine if:

- The discarded products today are reflective and behavioral (functional) even thought they are undesirable due to damage (not visceral),

- The usability of a product is determined solely by its visceral and behavioural elements, and not the reflective elements,

- The products with a reflective connection can be unusable, but are not discarded by the user.

The investigation also determined if a design intervention would have a significant change in the emotional response of the people towards discarded and unusable products. The design experiments are explained in detail in the next section.

\section{Research Through Design}

This investigation applied a research through design process which according to Frayling [4] is a scientific inquiry that uses the insights coming through the "practice of design' to give a better understanding of a complex world or context. Zimmerman, et al. [16]. concluded that this process is "where design researchers focus on making the right thing; artifacts intended to transform the world from the current state to a preferred state." In the current investigation the 'practice of design' were the experiments undertaken to improve the products; and these experiments were carried out to understand the reason behind products being discarded, or not. The practice of design helped in not merely thinking about what to make, but encouraged the designer to use the 'making', to create new insights. As illustrated in Figure 1, the stages in the research through design process did not happen in isolation, nor were they linear, instead these were interconnected with the other research methods, continuously being informed by the current state of the art literature, user group feedback, and also followed a cyclical direction due to the designer's own reflection in action [17].

The investigation used mixed methods; first, a usercentric approach collected pictorial evidence for discarded, usable and not discarded (but unusable) products from people living in the United Kingdom. Additionally, experts were involved in several stages of the investigation for fact checking and validation of the insights. Finally, the Kansei design principles were applied for evaluation of the finished products to confirm if the user could accept imperfection, and if design could extend the user-product connection. These are explained in detail in the next sections.

\subsection{User Research}

Open-ended interviews were conducted on 20 participants, and this was done via a Facebook group. Participants were asked to post pictures of furniture and narrate:

- Why the piece was still being used, if it was viscerally unappealing or lacked behavioural aspect,

- Why the piece was discarded, if it was viscerally well maintained and working well (behavioural),

- Why the piece was not discarded, even if it was viscerally damaged and/or lacked behavioural aspect.

This Facebook page collated the whole experience, feelings and relationship between the people and products from the beginning of purchase until the products were discarded/replaced. This helped envisage how people got emotionally attach to products and the reason behind 
people's thinking when they suggested that a product was undesirable. This stage allowed people to be more reflective regarding their reasons for discarding a product.

This information was put into the framework that identified the reason behind products deemed usable, despite the behavioural and visceral damage (Appendix 1). This revealed that the owners (users) perceived the broken parts as the more interesting parts, because these could tell a story of the journey, and experience of use for the product. The author interpreted these flaws as something to be celebrated and cherished because they made the furniture distinct from other mass produced products.

\subsection{Expert Interpretation}

Five experts, who either conducted similar investigation or worked in this field, were involved at various stages of the research through design process. Their expertise was gathered through one-on-one, open-ended interviews, which was later analysed using qualitative coding techniques [18]. These experts were,

1. Owner, second hand shop for furniture, UK,

2. Researcher, identification of the social value of products, UK,

3. Service designer, sustainable disposal of electronics and furniture, UK,

4. Professor in art, products as agents of discourse and public debate, UK,

5. Professor in communication design, UK.

The purpose behind involving these experts was to get valuable insight on how meanings evolved in discarded/ undesirable products. These insights confirmed important facts that informed the process of 'the design practice'. For example, experts 2 and 3 concluded that neither recycling nor up cycling was a solution for products that ended in a landfill. Later, expert 1 also supported the idea, and added that upcycling, to improve product use and appearance would not create a long-term change. His experience had led him to confirm that upcycling was a labour intensive, time consuming and a costly process with a huge probability of consumer's subjective disappointment. This particular instance of expert knowledge led to the decision of focusing on functionality (behavioural level) and physical look (visceral level) of the discarded products, rather than changing product identity (through recycling or upcycling).

1. Such expert interviews also allowed the designer to begin the experiments with certain informed presumptions,

2. Undesirable category - In case of discarded product, the informed presumption was that the products were discarded because of their loss of behavioural usefulness, which also resulted in them being viscerally low standard, and that there was no emotional importance associated with the product,

3. Desirable category - In case of usable product, if the product was being used, and in good visceral condition, then the informed presumption is that the product either had no visible damage and has an emotional importance associated to it by the user, or the product is new,

a. However, if the product is damaged (viscerally, behaviouraly), and still being used, the informed presumption is that the product must have a longterm emotional importance to the user,

4. Most desirable category- In case of unusable and not discarded products, if the product was unusable and not discarded then the informed presumption is that the product definitely has a long-term emotional importance attached to it.

\subsection{Experiment Design}

In total, seven experiments were undertaken on products that were in category 1,2 and 2(a), however, only 3 of those experiments are discussed in this paper. The 1st experiment was a pilot, which tested the design improvements, but did not involve any user for feedback. The next 2 experiments discussed applied the practice from the 1 st experiment, and also involved users before, during and after the experiments, and as a result the outcomes of the experiments were evaluated through a robust framework. Whilst the 2nd and the 3rd experiments showed a significant change in the emotional perception of the user towards the furniture, none of the experiments indicated otherwise.

The design experiments addressed the notion of 'unusable and broken products'; and, asked the question, can accessing usability (behavioural level) and embracing imperfection/impermanence of the found materials (visceral level) give meaning of mortality (reflective level) to category one (undesirable) furniture's?

Prior to the start of the experiments, the designer had to identify which category (undesirable, desirable and most desirable) the product would fit in before the experiments began. During the development of the experiments, whilst it was solely the designer who was involved in 'making', constant user interaction was encouraged to evaluate the transformation, in order to ascertain the emotional importance of the products due to the experiments. This enabled the designer to track any shifts in the users emotions towards the product, i.e., if the experiments were shifting the product from an undesirable category to the most desirable one. Finally, at the end, the transformation and 
the change in the emotional relevance of the products had to be evaluated by the user. The development and evaluation stage worked simultaneously to explore, improve and generate a better product.

The designer began these experiments with the mere notion that these products have a soul, and are more than just simply objects - there are stories that can only be told by the furniture that have been used on a day-to-day basis. The strategy for design was to combine Norman's [2] three levels of emotional design (visceral, behavioural, and reflective level) with Wabi Sabi philosophy that accepts the natural decay, and embrace its imperfection. Therefore, the main design principle was to highlight imperfection with the use of colour. This design principle embraced the view that broken and discarded furniture could be made beautiful and meaningful. It also mildly encouraged the notion that discarded furniture could be seen as meaningful, usable and unbroken.

The challenge was that each furniture provided a new context and was unique in their own way. Each furniture required having a unique approach towards creativity in design both in visceral and behavioural level that made each of them special. However, the same visceral design principal - white colour as the base, and brass colour as the accent of its imperfection and new materials - was repeated in every furniture to visually create the same message.

\subsection{Evaluation}

This study incorporated users at the heart of the experiments. Yoon, et al. [19]. concluded that designing products with an emotional fit requires an integrated approach in which design insights on user-product context is an essential part of the design activity. One such method is the Kansei approach, which was used to evaluate the response of users towards refurbished products, i.e., looks (eyes), smell, sound, feel (touch), and an overall good feeling. By showing the design idea and reparation process, participants (users) were given an opportunity to evaluate the history of the product, the change as a result of the experiments, and also the new redesigned furniture. This information was collected in form of a feedback form.

\section{Design Experiments: The Combination of Visceral, Behavioural, and Reflective Design}

The first experiment aimed at practicing the application of the identified design strategy and principles. This was done on a Mackintosh chair, a furniture masterpiece, however rendered undesirable, and currently in category 1 .

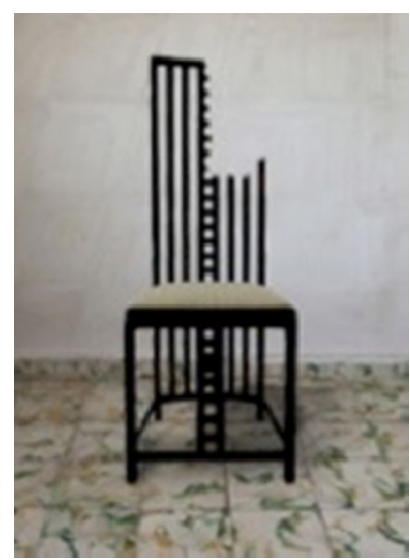

Figure 2 Charles Rennie Mackintosh

The second, is a category 2(a) product, i.e., a dinning set that is desirable and has the reflective and emotional elements, but lacked visceral elements.

The third, is a category 1 product, i.e., furniture that is undesirable and had no visceral, behavioural and reflective elements.

\subsection{Pilot Experiment: Popularised Chair}

This chair was found in a junk of a second-hand furniture shop. The owner remains unknown, however, the chair was actually designed by a famous Scottish architect, Charles Rennie [2]. Although it was broken, people may still recognise its initial renowned design (Figure 2).

Taking into account the informed presumption for this category of the product (1) the designer was able to explain that the chair was discarded because of its loss of behavioural usefulness, which also resulted in them being viscerally low standard (or vice versa). However, the most important element is that the chair had lost the emotional importance from its previous user.

\subsubsection{Reflective Design Solution}

For this product the developments were undertaken via a computer simulation, with the intention of actualization once the design was deemed suitable. Keeping in line with the chosen design principle's, the transformation of the chair was made with little intervention to preserve the chair's form that showed the essence of remains Mackintosh's design. Ching and Binggeli [13] suggested neutralized colour schemes as the most flexible one. Therefore, the development process started through refinishing the whole chair with white paint, and accentuating the broken backrest with gold brass color. This was done intentionally to highlight the imperfection of the chair, however the 


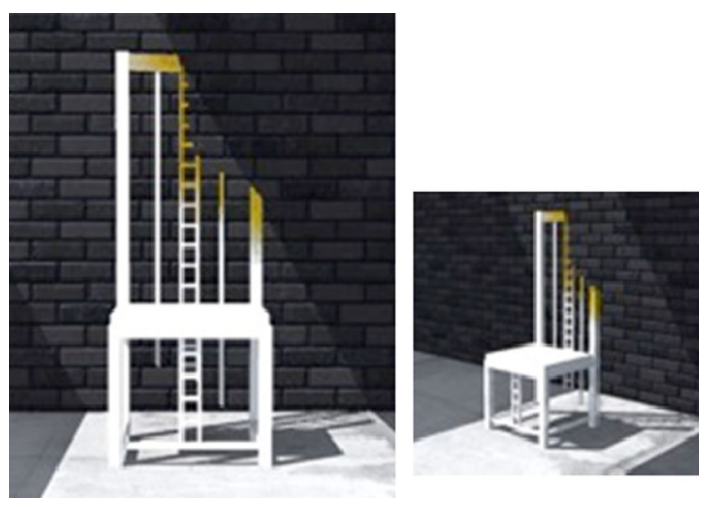

Figure 3 Drawing of the Makintosh's chair

golden colour made this aspect pleasing to the eye (visceral level) (Figure 3).

Whilst the defects in the chair were exposed and probably behaviouraly the chair might also be considered less comfortable to use, the identity of a Mackintosh's design (reflective design) was kept intact. The designer considered the real value of this chair not in its comfort of use (behavioural design) and its visual perfection (visual design), but in it being a masterpiece. However, the experiments could not ascertain if a user would have given the same importance to the chair's historical identity over comfort.

\subsubsection{Evaluation}

User evaluation for this chair was not possible within the time frame of this research. Nevertheless, it was highly acclaimed as a concept by the experts in Art (expert 3) and communication design (expert 4). The expert confirmed that the concept had the potential of re-popularising what was once famous and celebrated furniture, and gave it a second chance for usability.

The Kansei elements were also used to test if they were fit for the purpose of evaluation. The experts acclaimed the product highly on the basis of looks. However, suggested that a physical prototype would be a better medium to judge the elements of touch, sound and the overall feel of the product. The experts also agreed that the design principles were suitable to be carried out on all the product experiments, as these had a simple reoccurring message, of celebrating damage and decay.

\subsection{Experiment for Category 2a: Desired Category}

Linda and her family had been using their dining furniture set for over 20 years. They grew up with this table and this table helped carved the family memories every time they gathered together while having a family dinner. The dining set had never been replaced since the day the family bought it. This set of furniture would always keep the memory of
Linda's family and her childhood life. However, ageing made some of the material gradually weaken. The wood planks of the chair backrest were not able to hold the load they were starting to detach one by one, hence, the family could no longer use the chairs because it was dangerous to lean on. Also, the glass top of the table was cracked because of bearing a heavy burden. The family tried to cover the large crack by placing a tablecloth over the table. Linda and her family loved their dining set and they were willing to keep this table to the limit of its capability in accommodating the family activities (Figure 4).

The owner had a deep emotional importance attached to this dining set, and did not consider its poor usability and physical defects as inconvenience, hence the product's reflective elements were intact. However, Linda and her family had been avoiding using the chair because of the detached backrest, which made the chair unusable due to safety reasons, also the cracked glass made the table viscerally week.

Due to the emotional importance attached to the product, the challenge was to ascertain that the reparation does not transform the chair into something unfamiliar. Special consideration had to be given to preserve the original form and material of the furniture set that contained valuable memory and personality.

\subsubsection{Visceral Design Solution for the Chair}

For the chair the suggested design was to reconstruct the backrest by tying a rope as cordage to allow people to sit and lean comfortably (behavioural design). Again, the rigging rope was accentuated with gold colour to enhance its craftsmanship, and keep it in line with the agreed colour scheme (visceral design).

\subsubsection{Visceral Design Solution for the Table}

The design tried to cherish the crack and provoke a new perspective to the family by repainting the table in white and replacing the cracked glass with brass material (Figure 5). It showed contrast at a visceral level, enhancing the

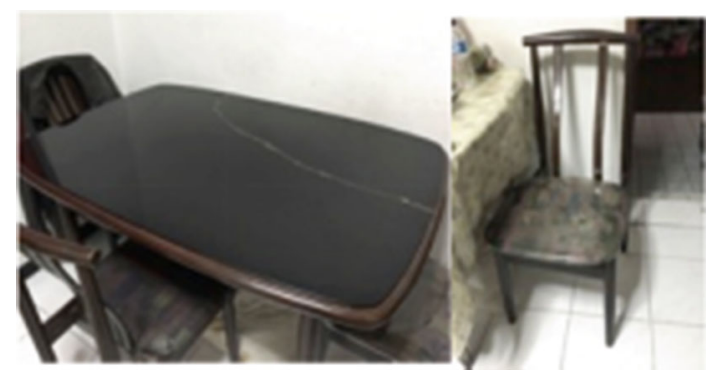

Figure 4 The broken family dinner table 


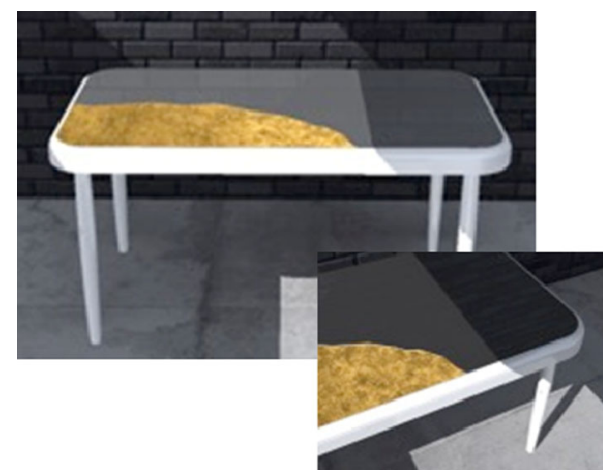

Figure 5 Drawings for the table reparation

shape of the crack rather than hiding it. Brass could create a beautiful patina in its ageing process that the family could cherish through its lifetime.

\subsubsection{Validation}

A user participant, who owned the "Childhood Chair" highlighted the quality of the suggested design, specifically in the method of refurbishing without too many interventions, consequently erasing the personality of the furniture. Based on the Kansei principles, the owner explained, "the colour makes the dining set more valuable than before. The process of reconstruction also does not change the old set, which means it does not erase my childhood memory." She rated the design concept high on look, touch, and colour based on the sample material, which was shown to her. However, evaluation on the overall good feeling could not be made at that point in time, as the product was not completed.

In maintaining the owner's childhood memory and her valuable emotional attachment, the original material of the chair and table was also preserved; only the missing parts were replaced with a new and distinctive material and colour to celebrate its imperfection. "For her, decay and broken part, as something that is "less valuable" could actually be an opportunity to become something that was "more valuable" - it was not something to be hidden, but to be celebrated.

\subsection{Experiment for Category 1: Undesired Product}

A discarded china cabinet was found from the junk of a second-hand furniture shop in Newcastle. The two missing side glasses made the cabinet less usable (behavioural level). Defects and scratches were found on many edges and this made it less desirable at a visceral level (Figure 6).

Therefore, keeping in mind the informed presumptions the designer was able to explain that the product had no

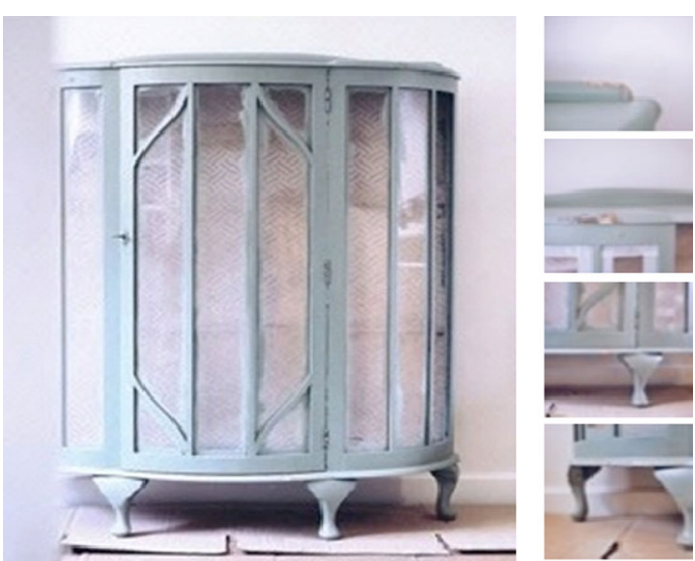

Figure 6 Discarded China cabinet

associated emotional importance, and it also did not have the visceral appeal nor did it have the behavioural elements left in it. Hence, the experiments had to give back the cabinet its functionality (behavioural elements), its visceral element, and as a result allow it to find a new meaning with a new user. Many potential users for the cabinet were involved throughout the experimentation, and also evaluated the outcome at the end.

\subsubsection{Visceral Design Solution}

As base colour, the overall main body of the furniture was painted in white, to represent a blank canvas for people to repaint it in future. The broken and missing parts of the furniture needed to stand out and change what people perceived as 'broken', undesired, into something that is 'unbroken', cherished and celebrated (Figure 7).

Defects and scratches were highlighted by contrasting gold colour to embrace its imperfection, creating a striking visual design, differentiating the new from the intact and 'undamaged' parts of the furniture's body.

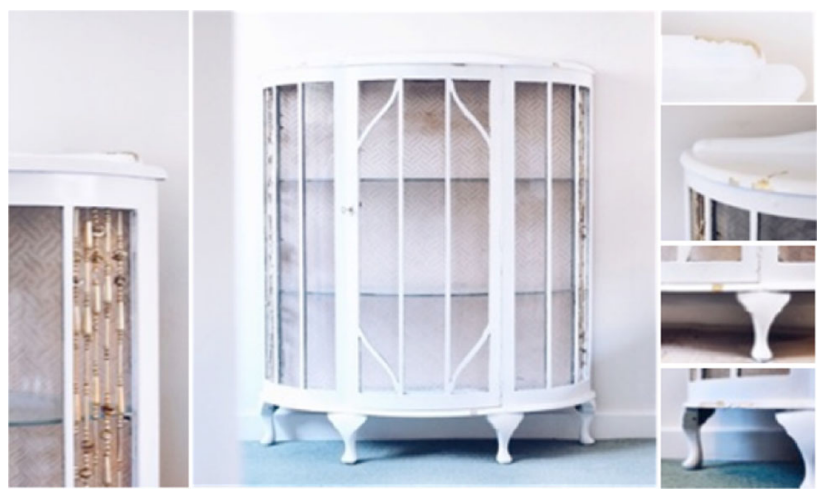

Figure 7 White base colour to provide a blank canvas 


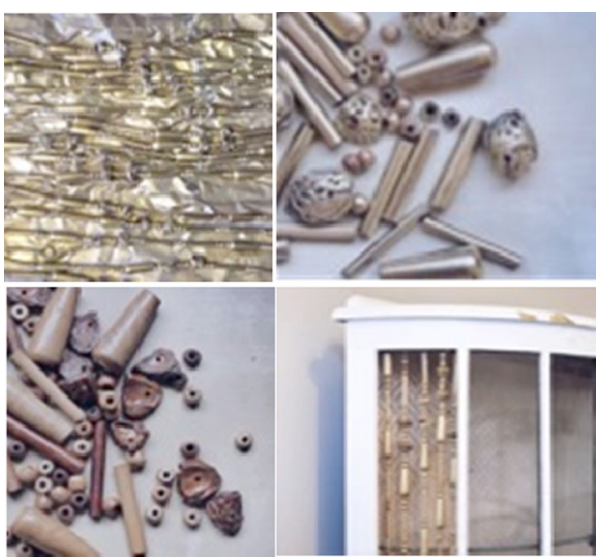

Figure 8 Golden beads as enhancement design

\subsubsection{Behavioural Design Solution}

The experience of use plays important role in enhancing a products behavioural elements, particularly in the case of furniture design. Discarded furniture should be designed and adapted with a good behavioural design for people to reuse them (purposefully). A successful behavioural design is when the discarded furniture can be reused well than how originally it was intended.

Initially, fabric materials replaced the two missing sides glasses. However, this didn't allow the users to show off the displayed objects inside the cabinet. Hence, another option was chosen. A set of discarded wooden beads were found from the junk of second-hand furniture shop, they were used as an alternative to cover the gap providing a new look to the cabinet, but at the same time maintaining the original purpose of the cabinet (Figure 8).

\subsubsection{Reflective Design Proposal: Embracing Imperfection}

The redesigned cabinet addressed the behavioural and visceral level of emotional design, however the long-term value could only be achieved through redesigning and reconstructing the perception of people towards products on a reflective level. Design on reflective level has a capability to deliver a message and provoke and engaged people to see discarded furniture in a different way, contemplate, rediscover value and recreate long-term empathy towards discarded products.

The design tried to deliver a message to potential users; the message was to celebrate the decaying process of the cabinet, find beauty in imperfection and impermanence, and see what is right in what is wrong. In simple words, the value of products does not transpire from a set of special objects, but it is determined by people's perception, experience, and attitude in accepting the natural change of products - both in positive or negative changes.
Through design interventions, as seen in the above-mentioned experiments, empathy, compassion, and positive changes of perception can be instilled, and reaction towards 'less valuable' product can be changed. Beyond usability and physical look, the cabinet was also seen as an object representing the experiences of use, and a potential user was seen to learn to embrace and admire the imperfections of this product.

\subsubsection{Evaluation}

The evaluation confirmed that the refurbished China cabinet conformed to the Kansei elements of look, touch, and sound. Additionally, the overall feel of the product was also highly acclaimed by the potential user. As a result of the design the emotional attachment, the user (in this case the person who bought the product) rated the refurbished cabinet high, specifically due to the idea of embracing the defects with gold, as subjugated through the Wabi Sabi approach.

\section{Findings}

The investigation found that most of users were emotionally attached to usability (behavioural level) and appearance (visceral level) of their products that are easily replaced. Most of the discarded and undesired products emerged when they were no longer usable or physically broken, defective and aged. It is important to point out that this did not represent the users desire to mend a broken product. Products were seen as tools for users to complete their task, rather than as living objects that have meaning, memory, personality and story.

The constant interaction with the users led to the finding that the key for a long lasting value of products lay with the 'people', and how they gave meaning to things. This confirmed Verganti's [20] comment, which can be summarised, as that meaning is not given by a designer, but by people, in this case the users themselves. Thus, in reflective design, designers can only propose the message to the users, and then the users have to interpret the message to give meaning, and create their own emotional significance of the product.

\subsection{Challenge with the Attachment to the Visceral Elements}

The findings suggested that today's satisfaction has a risk to become tomorrow's discard. User's attached to the visceral elements of the product were only able to maintain their products existence, as long as they felt, that the product's physical elements were intact. This was seen as the biggest weakness in case of discarded products, as the likelihood of change in aesthetical value of a product due to change in time, trends in fashion, cultural differences, ageing, decay, etc. is highly probable. 


\subsection{Challenges with the Attachment to the Behavioural Elements}

The findings also suggested that the user's attached to behavioural (functional) elements of the product will go after the new product with better usability, and performance rather than repair the existing product. In the current investigation many discarded products emerged from outdated behavioural design and ended up in the landfill - e.g., many electronic gadgets were replaced with more sophisticated technology gadgets, even if they still worked. The challenge is that products will evolve with better technology and capabilities, hence outperforming and reducing the value of the old one.

\section{Conclusions and Future Research Directions}

The investigation concluded the role of design in initiating a positive change, rather than merely making products that adapt to change. These positive changes were demonstrated whilst design was seen innovating new meaning for people through the experiments on undesirable and merely desirable products. The experiments adapted user's perception to use discarded products better (behavioural design) and visually enjoy it again (visceral design). However, the long term value from adapting discarded and undesirable products did not lie in redesigning and repurposing the artifacts to become usable and visually better, but in redesigning and reconstructing the perception of people towards these products (reflective level). These experiments demonstrated a level of positive reinforcement on the emotional connection between the product and the user, and were also seen increasing the lifespan of the products. Therefore, design was seen connecting functional and emotional elements of the product.

\section{Implication on Industry 4.0}

The challenge for designers and manufacturers is not in merely creating products, but in making people want them to be responsive towards their emotions and making them last for a long term. As a consequence of the investigation, a new problem space has emerged, which now needs to be seriously considered by those involved in the Industry 4.0 debates.

We spend a lot of time amongst products. Most of our memories are built and shaped by the products we use and interact with. Most of these memories are lost when a product is thrown away and discarded due to its visceral flaws. Whilst the visceral flaws of the product can be addressed with the experiments stated in this paper, the stories that go around the product are lost and never told. The hope is to make products more responsive and responsible towards human emotions and memories, and the challenge is to enable the products to capture and communicate these memories.
This consideration needs to be on the innovation of meaning', rather than mere innovation of products. Whilst the former enables people to live differently and better, the latter provides them the choices and a sense of independence. However, the problem of easy discard is huge, especially for the products that will be produced in the future. This is because now we are under immense pressure to re-create the way we live, build and act in the world. So, does digital design have a role to play in making the whole product experience and life cycle more reflective? Can digitization of products capture and store product memories for the purpose of increasing the emotional connection of the products? Could Ji-Won, et al. [21]. be applied to this as a solution? Could digitization allow the discarded products to communicate and empower people on a reflective level - especially in dealing with the real audiences/customers, in order for them to embrace furniture's journey and decay in a long term?

Open Access This article is distributed under the terms of the Creative Commons Attribution 4.0 International License (http://creative commons.org/licenses/by/4.0/), which permits unrestricted use, distribution, and reproduction in any medium, provided you give appropriate credit to the original author(s) and the source, provide a link to the Creative Commons license, and indicate if changes were made.

\section{Appendix 1}

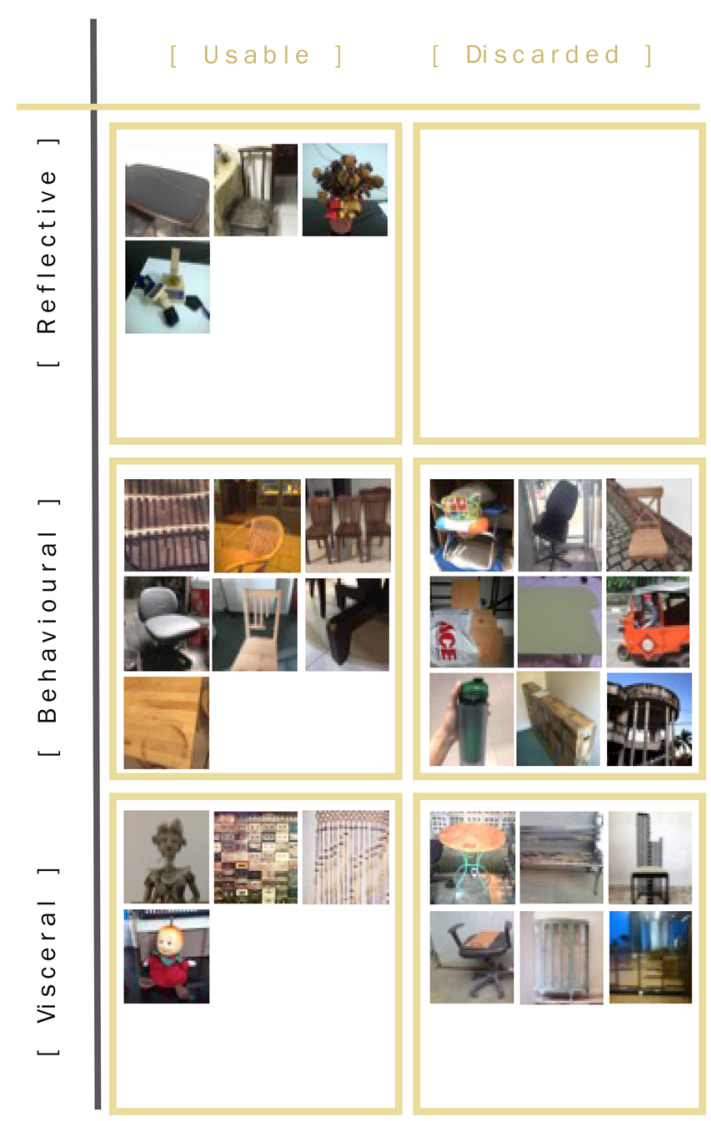




\section{References}

1. P Y Kwan. Exploring Japanese art and aesthetic as inspiration for emotionally durable design. Design Ed Asia Conference, http:// www.designedasia.com/2012/Full_Papers/Exploring\%20Japanese\% 20Art\%20and\%20Aesthetic.pdf. [Accessed 2016-03-14].

2. C R Mackintosh, Charles Rennie Mackintosh Society. http:// www.crmsociety.com/. [Retrieved 2016-05-01].

3. P W Jordan. Designing pleasurable products: An introduction to the new human factors. London: Taylor \& Francis, 2000.

4. C Frayling. Research in art and design. Royal College of Art, London. http://www.transart.org/wp-content/uploads/group-docu ments/79/1372332724-Frayling_Research-in-Art-and-Design.pdf. [Accessed 2017-05-28].

5. J Chapman. Emotionally durable design: Objects, experiences, and empathy. London: Routledge, 2015.

6. D A Norman. Emotional design: Why we love or hate everyday things. London: Hachette UK, 2007.

7. A Juniper. Wabi Sabi: The Japanese art of impermanence. North Clarendon: Tuttle Publishing, 2011.

8. S Brand. How buildings learn: What happens after they're built. London: Phoenix Illustrated, 1997.

9. S Walker. Sustainable by design: Explorations in theory and practice. London: Earthscan, 2006.

10. D Dabner, S Stewart, A Vickress. Graphic design school: The principles and practices of graphic design. Hoboken: John Wiley \& Sons, 2017.

11. A Sherin. Design elements: Color fundamentals. Massachusetts: Rockport Publishers, 2012.

12. D Kopec. Environmental psychology for design. 2nd ed. New York: Fairchild Publications, Inc, 2012.

13. D K Ching, C Binggeli. Interior design illustrated. 3rd ed. New Jersey: John Wiley \& Sons, Inc, 2012.

14. M Nagamachi, M A Lokman. Kansei innovation: practical design applications for product and service development. Florida: CRC Press, 2015.

15. A Gentner, C Bouchard, D E Elizondo, et al. (2012,). Kansei design approaches for the new concept development process. Paper presented at the Conference 2012, 5-6 July 2012, Venice.

16. J Zimmerman, E Stolterman, J Forlizzi. An analysis and critique of research through design: Towards a formalization of a research approach. Proceedings of the 8th ACM Conference on Designing Interactive Systems (DIS '10). ACM, New York, NY, USA, 310-319. http://dl.acm.org/citation.cfm?doid=1858171.1858228 [Retrieved on 2017-05-28].

17. D A Schön. The reflective practitioner: How professionals think in action. Basic Books, Inc., 1983.

18. V Braun, V Clarke, D GRAY, eds. Collecting qualitative data: A practical guide to textual, media and virtual techniques. http:// eprints.uwe.ac.uk/29936.

19. J Yoon, A Pohlmeyer, P Desmet. When 'feeling good' is not good enough: Seven key opportunities for emotional granularity in product development. International Journal of Design, http://www.ijdesign. org/ojs/index.php/IJDesign/article/view/2338, [2016-12-15].

20. M Morillo, C Dell'era, R Verganti. Exploring the role of 'outsider' interpreters in the development of design-driven innovations. International Journal of Technology Intelligence and Planning, 2015, 10(3-4): 222-253.

21. J W Song, D Norman, T J Nam, et al. Wireless device connection problems and desigh. Chinese Journal of Mechanical Engineering, 2016, 29(6): 1145-1156.

Mersha Aftab is a Senior Lecturer at Department of Design, Faculty of Arts, Design and Social Sciences, Northumbria University, UK since 2012. In 2013 she was awarded her doctorate on the topic 'design as a functional leader: a case study to investigate the role of design as a potential leading discipline in multinationals'. Mersha's work has led her to collaborate with Philips, Nokia, Daimler, Sony Erricson, Lego, Google and Samsung. Her interest lies in exploring the role of design in triggering a change in the culture of organisations. E-mail: mersha.aftab@gmail.com

Helen Agustin Rusli, is an interior designer/Architect based in Indonesia. She is the founder of an interior design consultancy named SenimanRuang. She pursued her Masters at Northumbria University, $U K$. Her research interests lies in creating a theoretical framework for making products last longer and also developing new concepts like adoptive design for interiors. She is currently implementing these concepts within her company. E-mail: agustine.helen@gmail.com 